Ann. Zootech., I969, 18 (4), 385-398.

\title{
VALEUR ALIMENTAIRE DU MANIOC ET DE DIFFÉRENTES CÉRÉALES DANS LES RÉGIMES DE SEVRAGE PRÉCOCE DU PORCELET : UTILISATION DIGESTIVE DE L'ALIMENT ET EFFET SUR LA CROISSANCE DES ANIMAUX
}

\author{
A. AUMAITRE \\ avec la collaboration technique de M. KIEHL et Huguette Dewulf \\ Station de Recherches sur l'Élevage des Porcs, \\ Centre national de Recherches zootechniques, 78 -Jouy-en-Josas \\ Institut national de la Recherche agronomique
}

\section{SOMMAIRE,}

La nature du constituant de base (céréales, manioc) incorporé à la ration de sevrage précoce du porcelet, influence de façon notable les performances des animaux. Ainsi, nous avons observé une croissance plus élevée avec le manioc et l'orge qu'avec le blé ou le maís. Les différences peuvent être expliquées en partie par un effet particulier sur l'état sanitaire des animaux et sur la digestibilité globale de la ration.

L'utilisation digestive apparente de tous les éléments de la ration varie suivant la nature de la céréale : ainsi, contrairement à ce qu'on observe chez le Porc plus âgé, l'orge, le blé et le maïs présentent une digestibilité comparable dans le cas où l'aliment est distribué en quantité égale. Le manioc améliore la digestibilité de 4 à 5 p. roo par rapport au blé et diminue la fréquence des diarrhées aussitôt après le sevrage. Les résultats montrent que le blé, l'orge, le maïs et l'avoine décortiquée présentent pour le porcelet une valeur en énergie digestible comparable alors que le manioc est plus riche.

\section{I. - INTRODUCTION}

La ration alimentaire du Porc est essentiellement constituée de céréales ou de produits amylacés. Le sevrage consiste à supprimer progressivement aux jeunes porcelets le lait de leur mère, et à le remplacer par un aliment concentré. Or, le jeune, que ce soit 1'Enfant, le Vea1 ou le Porcelet, refuse de consommer de grandes quantités d'aliment concentré et l'on pense généralement que son tube digestif n'est pas en mesure de l'utiliser. Ceci constitue l'obstacle le plus sérieux à l'application en élevage 
des techniques de sevrage précoce. On sait, de plus, que les organes digestifs du porcelet allaité sécrètent peu d'amylase (LEWIS et al., I955; WALKER, I959), mais qu'il se produit une adaptation de 1'animal au régime contenant de l'amidon (HARTMANN et al., I959; Aumaitre et al., I964).

De plus, suivant l'espèce végétale dont il provient (RADI,EY, I953) (PÉRISSE et al., I956; GuilBo't et al., I962 ; Aumaitre, I966), 1'amidon est hydrolysé plus ou moins vite par les enzymes. Enfin, on sait que l'origine de l'amidon peut influencer de façon notable la digestibilité apparente ế l'utilisation métabolique des autres éléments de la ration de l'Homme (LANGwORTHY et DEUEL, I920), du Rat (PÉRISSE et Jacquot, i956), du Porc en croissance (Madsen, i963, Homb et Lyso, I964). Ces dernières indications peuvent orienter le choix vers tel ou tel type d'amidon pour constituer un régime de sevrage, d'autant que l'amidon non dégradé dans l'intestin grêle du nourrisson peut conduire à une diarrhée fermentaire dont les répercussions pathologiques ont été analysées par WEIJERs et al. (I965).

Nous avons tenté au cours de deux expériences, de déterminer la valeur alimentaire et l'utilisation digestive de régimes contenant des quantités comparables d'amidon de sources différentes (céréales, manioc) dans les régimes du porcelet sevré à 5 semaines. En effet, si les diverses céréales (avoine décortiquée, blé, orge et maīs) sont généralement utilisées pour le sevrage précoce du porcelet (CARROI, I, I942; Smith et LuCAS, I957; SALMON-LEGAGNEUR et Février, I959), le manioc semble absent de tels régimes. Seuls Anonyme (I962), Hansen (I964) et Homb et Lysö (I964), recommandent de 1'utiliser jusqu'à 40 p. Ioo de la ration du Porc, alors que les propriétés et la valeur alimentaire de la farine de manioc sont connues chєz le porcelet ('TERROINE I93I) et qu'il est très bien utilisé par l'Homme à l'état cru (PÉrisse et al., I956).

Nous avons ainsi étudié l'effet de l'incorporation de différentes céréales ou de manioc à $5^{\circ} \mathrm{p}$. Ioo environ du régime sur le comportement des porcelets sevrés à 5 semaines et placés dans des conditions pratiques d'élevage. Nous avons enfin étudié l'influence de la nature du constituant de base du régime sur la digestibilité apparente des éléments de la ration pour des animaux alimentés à volonté ou rationnés et placés en cage à métabolisme.

\section{II. - MATÉRIEL E'T MÉTHODES}

\section{Animanx}

Expérience I. - I 14 porcelets de race Large White Yorkshire provenant de 24 portées de notre élevage expérimental ont été utilisées. Au sein de chaque portée, 3 lots de 2 animaux de même poids ( $\mathrm{I}$ mâle et I femelle par lot) ont été constitués suivant la méthode des blocs.

Les animaux recevant la ration à base de blé constituaient pour chaque portée le lot témoin. On a effectué ainsi I 2 répétitions de 2 animaux par traitement pour comparer, au sein d'une portée soit les régimes blé, maïs, avoine décortiquée, soit les régimes blé, orge, manioc.

Expérience $I I .-4^{\circ}$ porcelets provenant de 8 portées ont été placés en cage de digestibilité circulaire (JouANDEI et al., 1964) équipées de plateaux en polyvinyle et étudiées pour permettre la récolte séparée des urines et des fèces sur les animaux des deux sexes. Le local est climatisé à $28^{\circ} \mathrm{C}$ et $60 \mathrm{p}$. Ioo d'humidité relative.

Les animaux de poids identiques étaient affectés au hasard aux différents traitements. Après une semaine d'adaptation, on mesurait la digestibilité des aliments en égalisant les quantités d'aliment ingérées entre les lots sur 3 périodes successives de 6 jours sans interruption. 


\section{Aliments}

La ration de sevrage contenait environ $50 \mathrm{p}$. too de céréales ou de manioc de Madagascar en racines (tabl. I). Elle apportait de plus dans chaque cas une quantité égale de supplément protidique représentant les $2 / 3$ de l'apport azoté total et l'on réalisait des régimes isoazotés en supplémentant ou non avec du gluten de blé.

TABLEAU I

Composition des régimes expérimentaux (p. Iоo)

\begin{tabular}{|c|c|c|c|c|c|}
\hline Régime & Blé & $\begin{array}{c}\text { Avoine } \\
\text { décortiquée }\end{array}$ & Mais & Orge & Manioc \\
\hline 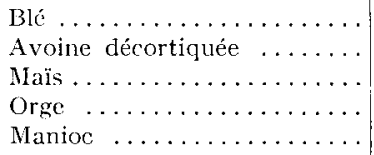 & 51,5 & 52,0 & 48,5 & 49,5 & $1 / 4,5$ \\
\hline $\begin{array}{l}\text { Gluten de blé............ } \\
\text { Ḿclange protidique }{ }^{(1)} \ldots \ldots \\
\text { Oxyde de chrome }\left(^{(2)} \ldots \ldots \ldots\right.\end{array}$ & $\begin{array}{r}0,5 \\
17,5 \\
0,5\end{array}$ & $\begin{array}{l}0 \\
47, ; \\
0, ;\end{array}$ & $\begin{array}{r}3,5 \\
17,5 \\
0,5\end{array}$ & $\begin{array}{r}2,5 \\
47,5 \\
0,5\end{array}$ & $\begin{array}{r}7,5 \\
47,5 \\
0,5\end{array}$ \\
\hline
\end{tabular}

(1) Composition p. 100 : tourteau de soja : 12,5. Sucre : 20,8. Huile d'arachide : 12,5. Farine de poisson : 1',6. Levure : 10,5. Poudre de lait érrémé : 20,8. Minéraux : 5,2. Vitamines, antibiotiques : 3,1.

(2) L'oxyde de chrome était introduit en remplacement d'une quantité équivalente de sucre dans la $1^{\text {re }}$ expérience seulement.

TABLEAU 2

Caractéristiques analytiques des produits de base et des aliments utilisés dans l'expérience ( $p .100$ du produit frais)

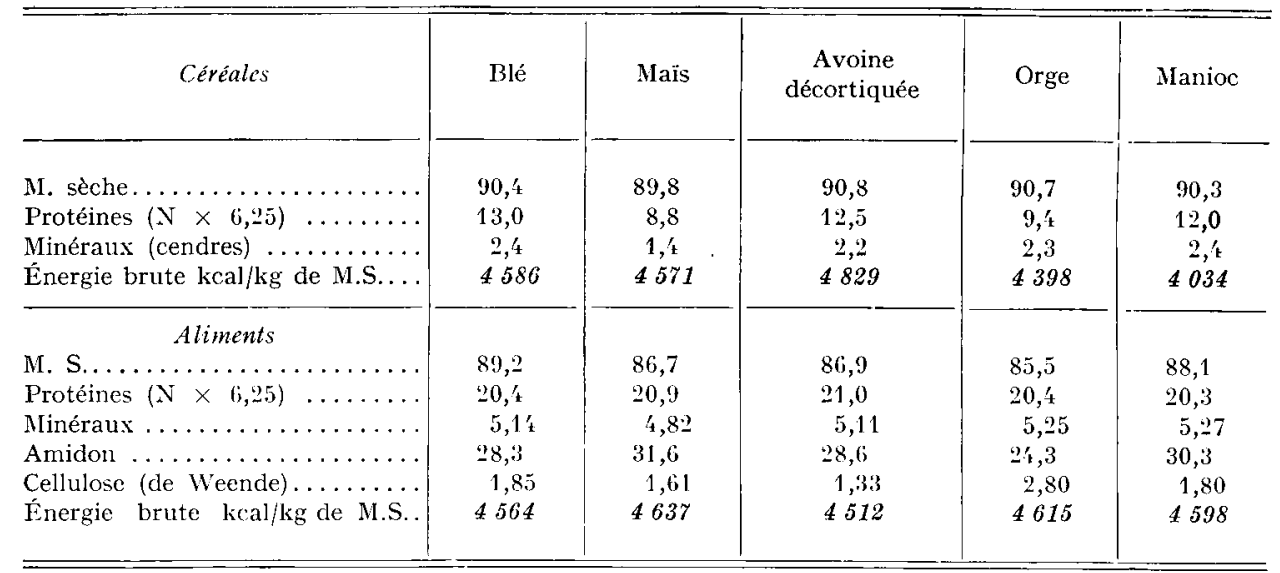


Les aliments étaient granulés à $2,5 \mathrm{~mm}$ de diamètre et distribués à volonté dans des petits distributeurs automatiques. En vue de contrôler la digestibilité des principaux éléments de la ration offerte à volonté (matière sèche, protéines, minéraux), $0,5 \mathrm{p}$. Ioo d'oxyde de chrome ont été ajoutés dans les aliments (exp. I).

La composition chimique des produits de base et des aliments fabriqués a été déterminée (tabl. 2). Ils contiennent environ $20 \mathrm{p}$. Ioo de protéines brutes et 4 mégacalories d'énergie brute par kg. Leur teneur en amidon vrai est comprise entre 25 et 30 p. 100.

\section{Mesures effectuées}

Pour chaque lot expérimental, pendant un mois après le sevrage ( 35 au $63^{\mathrm{e}}$ jour), le gain de poids individuel des animaux et la quantité d'aliment consommé (pour deux animaux) ont été mesurés chaque semaine. Les animaux placés en cage à bilan ont été pesés au début et à la fin de chaque période. L'efficacité alimentaire moyenne de la ration ainsi que les coefficients d'efficacité protidique et énergétique ont été calculés pour l'ensemble de la période.

L'état sanitaire des animaux a été noté pour chaque porcelet et les résultats exprimés en nombre de jours de diarrhée déterminés de visu, rapportés par porcelet et par période expérimentale.

Pour la détermination de la digestibilité apparente des éléments de la ration à l'aide du marquage à l'oxyde de chrome, la collecte de fèces a été effectuée deux fois par jour pour chaque lot. Le séchage a été réalisé immédiatement à $60^{\circ} \mathrm{C}$ pendant 24 heures; un échantillon moyen par semaine a été ensuite réalisé par lot pour toute la durée de l'expérience.

Sur Jes animaux placés en cage de digestibilité, les prélèvements de fèces effectués quantitativement deux fois par jour sont conservés sous acide sulfurique N/ro, broyés et échantillonnés ainsi que l'urine, collectée sous acide.

L'azote total a été dosé par la méthode de Kjeldahl, l'énergie des aliments est déterminée à la bombe calorimétrique balistique. L'amidon vrai est dosé après autoclavage et dégradation enzymatique par la glucamylase d'après la quantité de glucose libérée (glucose oxydase) par la méthode de Thivend et al. (I965). Le dosage de l'oxyde de chrome est réalisé après fusion alcaline et transformation en bichromate, par iodométrie.

\section{Interprétation des résultats}

La signification des différences observées a été testée à l'aide de la comparaison multiple des moyennes par rapport à un même témoin selon la méthode de DunNETT (1964).

III. - RÉSULTATS

\section{A. - Expériences $I$ : animaux nourris à volonté}

Les performances des animaux recevant le régime à base de blé ont été identiques dans les deux comparaisons, aussi les résultats sont rassemblés en rapportant une seule fois les performances des porcelets considérés comme témoins.

\section{Quantités d'aliments consommés.}

Les quantités moyennes d'aliments consommés varient dans des proportions notables suivant les lots. La consommation maximum est observée pour le manioc (tabl. 3) où l'amélioration atteint I3 p. Ioo de la quantité mesurée pour le régime témoin. L'évolution de la quantité consommée par jour (fig. I) nous montre que les différences apparaissent dès le sevrage pour l'orge et le manioc, alors que l'avoine décortiquée provoque une ingestion supérieure, 2 semaines seulement après le sevrage; pour le mais, les quantités consommées évoluent de façon identique au lot témoin à base de blé. 


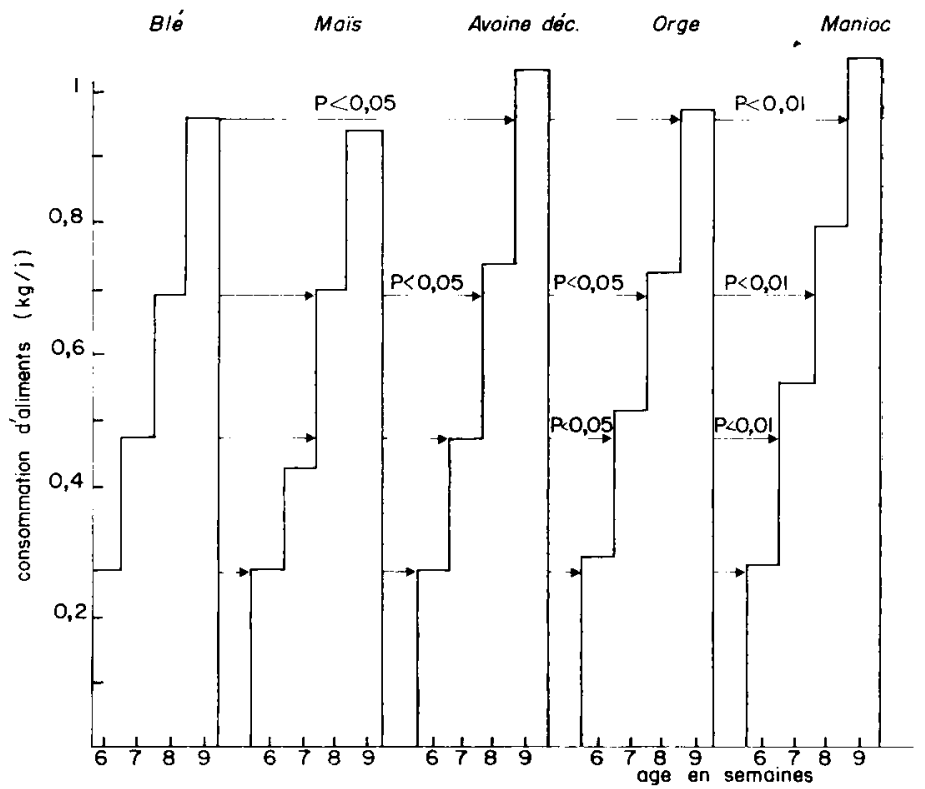

FIG, I, - Évolution suivant l'âge de la quantité d'aliment consommée par les porcelets. nourris à volonté suivant la nature du constituant de base ( $\mathrm{P}<\mathrm{o}, \mathrm{or}$ indique la signification des différences observées entre le régime à base de blé et le régime expérimental)

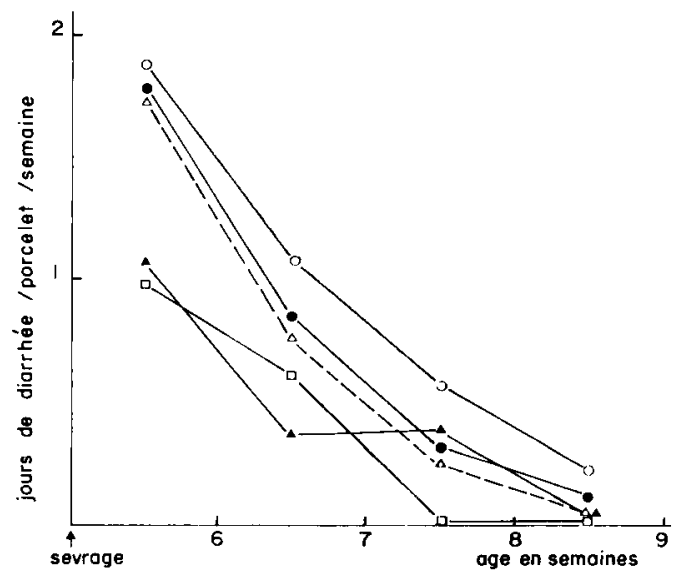

FIG. 2. - Etat sanitaire des animaux: fréquence des diarrhées selon l'âge et la nature du régime

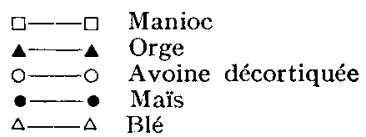


2. État sanitaire des animaux.

La fréquence moyenne de l'état diarrhéique est très différente suivant les régimes (fig. 2).

La fréquence maximum est observée au cours de la semaine qui suit le sevrage. Les différences qui apparaissent entre les lots montrent qu'il existe des régimes qui limitent la diarrhée (manioc, orge) et des régimes qui la favorisent (blé, maïs et surtout avoine décortiquée). La diminution constante de la fréquence au cours de la période s'objerve pour tous les régimes mais elle est plus rapide pour le manioc que pour tous les autres lots. Par contre, l'avoine décortiquée entretient un état diarrhéique persistant pour certains animaux.

\section{Digestibilité de la ration.}

Les valeurs moyennes des coefficients de digestibilité apparente varient dans des proportions importantes avec le régime (tabl. 3). C'est ainsi que la digestibilité apparente de la matière sèche, des protéines et des cendres des rations à base d'avoine ou de maïs est plus faible que pour le blé et l'orge. A l'opposé, la digestibilité du régime à base de manioc est plus élevée que celle des autres rations pour tous les éléments considérés.

TABLEAU 3

Quantités d'aliment consommées et digestibilité des différents éléments suivant le régime distribué à volonté

(Expérience I)

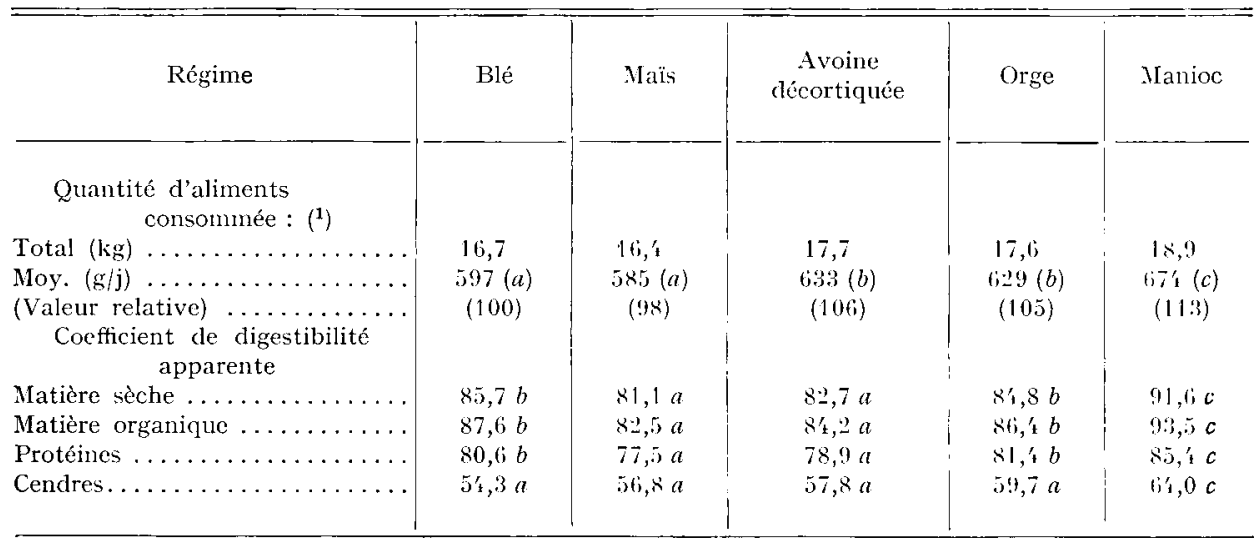

(1) Les valeurs affectées de la même lettre ne sont pas significativenent différentes entre elles.

On peut souligner, en effet, que la différence entre les coefficients observés pour le meilleur régime (manioc) et le moins bon (maïs) atteint Io p. Ioo pour la matière sèche et $5 \mathrm{p}$. Ioo pour les protéines.

De plus, on constate une augmentation des différents coefficients avec l'âge des animaux quel que soit le régime étudié. Entre la $3^{\mathrm{e}}$ et la $4^{\mathrm{e}}$ semaine d'expérience ( 8 à 9 semaines d'âge) on observe un plateau correspondant à 84 p. Ioo pour la matière sèche et $82 \mathrm{p}$. Ioo pour les protéines de la ration (fig. 3), les valeurs observées pour les minéraux présentent la même évolution dans le temps. 


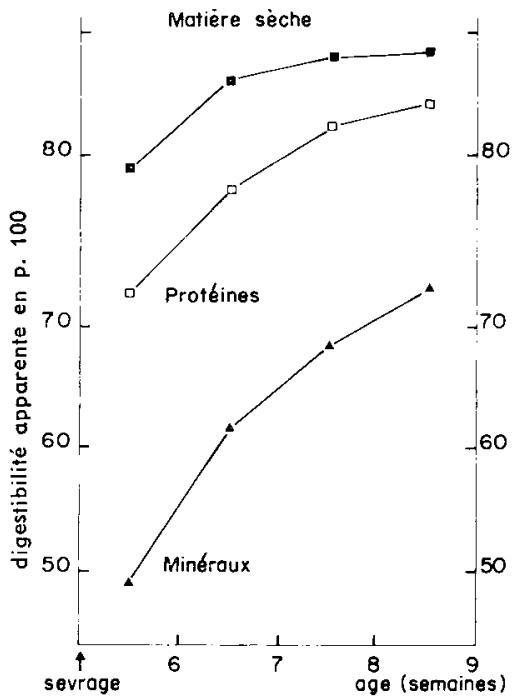

Fig. 3. -- Évolution des coefficients de digestibilité apparente de quelques éléments de la ration suivant l'áge des porcelets (Expérience I)

\section{Vitesse de croissance.}

Les croissances pondérales des deux lots d'animaux témoins (régime à base de blé) sont identiques dans les deux comparaisons ( 360 et $365 \mathrm{~g}$ de gain/jour). Les résultats moyens sont présentés globalement pour les 4 régimes expérimentaux par rapport au blé considéré comme témoin (tabl. 4). Le poids moyen des porcelets en

\section{TABI,EAU 4}

Vitesse de croissance des porcelets et efficacité des différents régimes

(Expérience II)

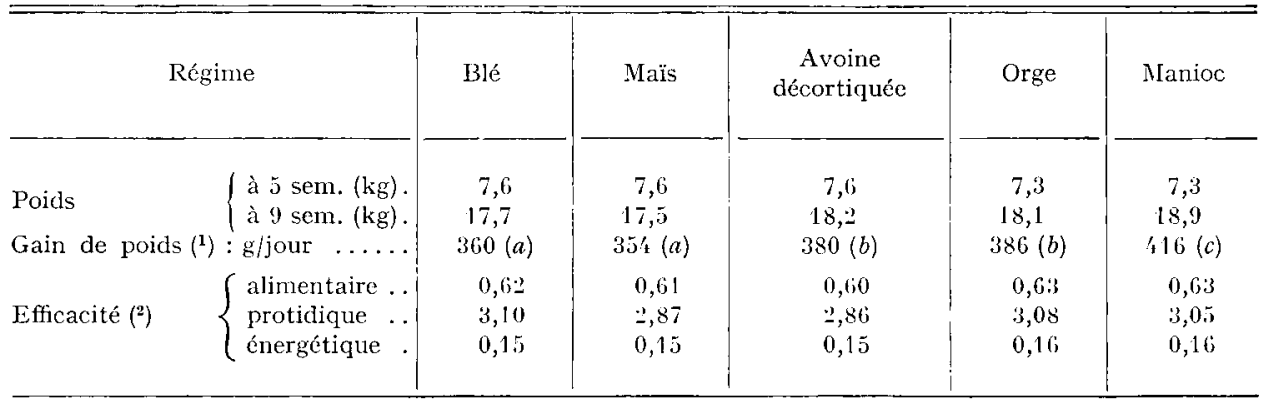

(1) Les valeurs affectées à la même lettre ne sont pas significativement différentes entre elles.

(2) Aucune des valeurs trouvées n'est significativement différentes des autres.

Efficacité alimentaire : $\mathrm{kg}$ de gain de poids/kg d'aliment consommć.

Efficacité protidique : $\mathrm{kg}$ de gain de poids/kg de protéines consommées.

Efficacité énergétique : $\mathrm{kg}$ de gain de poids/Mégacalorie d'aliment consommé. 
fin d'expérience ainsi que les gains de poids calculés sont rapportés au tableau 3. On constate que pour la période suivant le sevrage, le gain de poids varie suivant les régimes : les animaux recevant les rations à base de manioc, d'orge et d'avoine décortiquée ont une vitesse de croissance de 5 à I 5 p. Ioo supérieure à celle du témoin (blé). L'augmentation du poids moyen à 9 semaines atteint $\mathrm{I}, 6 \mathrm{~kg}$ pour le manioc, on l'observe dès la première semaine pour les meilleurs régimes.

\section{Efficacité des aliments.}

L'efficacité alimentaire observée est élevée puisque I $\mathrm{kg}$ d'aliment permet un gain de poids de 600 à $630 \mathrm{~g}$ en moyenne (tabl. 4). On ne constate cependant pas de variation significative avec le régime. Ce phénomène se retrouve au niveau de l'efficacité protidique globale, légèrement plus faible dans les lots à base de maîs et d'avoine décortiquée. L'efficacité énergétique varie également assez peu, elle est cependant supérieure pour les régimes qui provoquent la vitesse de croissance la plus forte (orge et manioc). La valeur moyenne de l'efficacité de l'aliment varie également avec l'âge; elle présente un maximum trois semaines après le sevrage puis diminue.

\section{B. - Animaux alimentés en quantité égale et en cage de digestibilité (Expérience II)}

\section{Utilisation digestive des différents éléments de la ration.}

La digestibilité de la matière sèche, des protéines et de l'énergie de la ration est en général supérieure au lot Témoin pour les régimes à base d'avoine décortiquée et de manioc. Cependant, seuls les résultats obtenus avec le manioc sont statistiquement différents de ceux observés pour le lot Témoin pour tous les éléments de la ration (tabl. 5).

\section{TABLEAU 5}

Coefficients de digestibilité apparente des différents éléments de la ration: éléments digestibles des différentes rations

(Expérience II)

\begin{tabular}{|c|c|c|c|c|c|}
\hline Régime & Blé & Maïs & $\begin{array}{c}\text { Avoine } \\
\text { décortiquée }\left({ }^{2}\right)\end{array}$ & Orge & Manioc \\
\hline Digestibilité (1) & & & & & \\
\hline M. S. .................. & $8^{\prime}, 3 a$ & $85,8 a$ & $87,4 a b$ & $8^{\prime}, 0 a$ & $89,1 \quad b$ \\
\hline Protéines..$\ldots \ldots \ldots \ldots \ldots \ldots$ & $83,6 a$ & $85,0 a$ & $85,7 a$ & $83,+a$ & $87,6 b$ \\
\hline 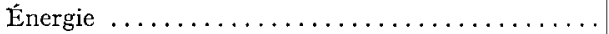 & $87,1 a$ & $87,3 a$ & $89,2 a$ & $85,7 a$ & $90,6 b$ \\
\hline Cellulose.$\ldots \ldots \ldots \ldots \ldots \ldots \ldots$ & $39,9 a$ & 51,$6 ; b$ & $4_{1}^{\prime}, \geq a$ & $41, \overline{5} a$ & $67, '+c$ \\
\hline Éléments digestibles de la ration & & & & & \\
\hline Énergie digestible $\mathrm{kcal} / \mathrm{kg}$ M.S. . . . . . . . . & $3973 a$ & $4046 a b$ & 's 02 's $a$ & $3955 a$ & $t_{1} 185 b$ \\
\hline Protéines digestibles g/kg M.S. . . . . . . . . . & $189 a$ & $207 a$ & $207 a$ & $199 a$ & $202 a$ \\
\hline
\end{tabular}

( $\left.{ }^{1}\right)$ Valeurs moyennes, 8 porcelets par régime et 3 périodes ininterrompues par porcelet de 42 à 60 jours

(2) Récolte impossible sur une période complète pour 2 porcelets (diarrhée). 
Ainsi les résultats obtenus permettent de calculer les valeurs des éléments digestibles des rations. On peut noter que la ration à base de manioc renferme une quantité d'énergie digestible supérieure à tous les autres régimes. De même, on peut souligner que les régimes à base de blé et d'orge ont la même énergie digestible.

Les coefficients de digestibilité des principaux éléments ont été comparés en fonction du sexe des animaux, indépendamment de la nature des régimes (tabl. 6). On constate que les résultats moyens pour les trois périodes considérées sont identiques pour les mâles castrés et les femelles, pour la matière sèche, l'énergie et les protéines des rations. Enfin, les coefficients de variation intra-groupe de la digestibilité apparente calculés pour les 8 animaux de chaque lot sont semblables pour tous les régimes : ils sont d'environ $3 \mathrm{p}$. Ioo pour les protéines et $2,2 \mathrm{p}$. Ioo pour l'énergie.

Les coefficients de rétention azotée varient dans des proportions limitées suivant le régime (tab1. 7). Seule l'avoine décortiquée entraîne une rétention azotée significativement supérieure à tous les autres régimes. Il existe également pour le régime à base de mais une tendance à la diminution de la rétention azotée par rapport aux autres groupes $(\mathrm{P}<0, \mathrm{IO})$. On observe enfin une variation avec l'âge du coefficient de rétention azotée : il est de $60 \mathrm{p}$. Ioo entre 40 et 46 jours et de $64 \mathrm{p}$. Ioo environ entre 47 et 60 jours.

Ėnfin, il n'existe aucune différence notable entre le pourcentage de protéines par rapport au gain de poids quelle que soit la nature du régime.

TABLEAU 6

Utilisation digestive de la ration : variations dues au sexe

\begin{tabular}{|c|c|c|c|c|c|}
\hline \multirow{2}{*}{ Sexe } & \multirow{2}{*}{$\begin{array}{c}\text { Nombre } \\
\text { d'observations }\end{array}$} & \multicolumn{3}{|c|}{ Digestibilité } & \multirow{2}{*}{ 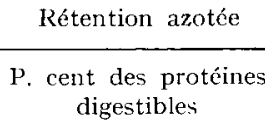 } \\
\hline & & MI.S. & Protéines & Énergie & \\
\hline Mâles & 里 & 84,3 & 85,1 & 87,1 & 63,5 \\
\hline Femelles $\ldots \ldots$. & 21 & $8 /, 3$ & 84,7 & 87,7 & 61,8 \\
\hline
\end{tabular}

TABLEAU 7

Coefficient de rétention azotée suivant les régimes: valeurs relatives aux protéines digestibles et au gain de poids

\begin{tabular}{|c|c|c|c|c|c|}
\hline Régime & Blé & Maïs & Avoine & Orge & Manioc \\
\hline $\begin{array}{r}\text { Rétention azotée p. } 100 \text { des protéines } \\
\text { digestibles } \ldots \ldots\end{array}$ & 62,3 & 59,2 & $66,2(1)$ & 62,5 & $6 \geq x^{\prime}$ \\
\hline Protéines p. 100 du gain de poids & 15,8 & 15,8 & 15,1 & 15,1 & 16,0 \\
\hline $\begin{array}{c}\text { Protéines retenues } \mathrm{g} / \mathrm{kg} \quad \mathrm{M} . \mathrm{S} . \text { de la } \\
\text { ration } \ldots \ldots \ldots \ldots \ldots \ldots \ldots \ldots \ldots \ldots \ldots \ldots\end{array}$ & 118 & 12: & 137 & 121 & 126 \\
\hline
\end{tabular}

(1) Valeur significativement différente de toutes les autres $\mathrm{P}<0,0$. 


\section{Croissance pondérale et efficacité de l'aliment.}

Les valeurs moyennes du gain de poids pour les trois périodes sont présentées selon le régime au tableau 8 . Pour une quantité égale d'aliment reçue, on constate une vitesse de croissance plus élevée pour les animaux qui reçoivent les rations à base d'avoine décortiquée, d'orge et de manioc. Parallèlement, les efficacités alimentaires et protidiques sont meilleures dans les mêmes lots par rapport au témoin à base de blé. L'efficacité énergétique varie; elle est significativement différente de celle du blé seulement pour le régime à base de manioc.

\section{TABLEAU 8}

Croissance des animaux, quantités moyennes d'aliment et d'eau consommées et état sanitaive; efficacité alimentaire comparée pour les animaux rationnés (1)

(Expérience II)

\begin{tabular}{|c|c|c|c|c|c|}
\hline Régime & Blé & Maïs & $\begin{array}{c}\text { Avoine } \\
\text { décortiquée }\end{array}$ & Orge & Manioc \\
\hline $\begin{array}{c}\text { Gain de poids g/jour } \ldots \ldots \ldots \ldots \ldots \\
\text { Quantité consommée g/jour : }\end{array}$ & $353 a$ & $313 a$ & $388 b$ & $387 b$ & $405 b$ \\
\hline Aliment $\ldots \ldots \ldots \ldots \ldots \ldots \ldots$ & $50^{\prime}$ & 500 & $50 \%$ & 506 & 506 \\
\hline Eau $\ldots \ldots \ldots \ldots \ldots \ldots \ldots \ldots \ldots \ldots \ldots$ & $2215 a$ & $1962 b$ & $215+a$ & $1723 b$ & $\geq 102 a$ \\
\hline $\begin{array}{l}\text { Diarrhées (jour) Porcelet } \ldots \ldots \ldots \ldots \\
\text { Efficacité des aliments }\left({ }^{2}\right)\end{array}$ & $2,5 \quad a$ & $2,5 \quad a$ & $2,5 \quad a$ & $1,7 \quad b$ & $1,5 \quad b$ \\
\hline $\begin{array}{l}\text { Alimentaire } \ldots \ldots \ldots \ldots \ldots \ldots \ldots \ldots \ldots \\
\text { Protidique } \ldots \ldots \ldots \ldots \ldots \ldots \ldots \ldots \\
\text { Énergétique } \ldots \ldots \ldots \ldots \ldots \ldots \ldots\end{array}$ & $\begin{array}{l}0,69 a \\
3,40 a \\
0,16 a\end{array}$ & $\begin{array}{l}0,69 a \\
3,31 a \\
0,16 a\end{array}$ & $\begin{array}{ll}0,77 & b \\
3,81 & b \\
0,17 & a\end{array}$ & $\begin{array}{l}0,76 b \\
3,59 a \\
0,17 a\end{array}$ & $\begin{array}{ll}0,80 & b \\
3,94 & b \\
0,20 & b\end{array}$ \\
\hline
\end{tabular}

( $\left.{ }^{1}\right)$ Valeur moyenne pour 8 porcelets pendant 3 périodes de 6 jours.

(2) Unités au tableau 4.

Enfin, la fréquence des diarrhées est plus faible dans les régimes à base de manioc et d'orge : les valeurs sont statisquement différentes du lot témoin et des deux autres régimes.

\section{DISCUSSION}

\section{Utilisation digestive des régimes}

Le point le plus important dans nos résultats semble être que dans les deux expériences, le blé et l'orge provoquent une digestibilité globale absolument comparable de tous les éléments du régime. Ces résultats sont contraires à ceux trouvés par de nombreux auteurs sur le Porc plus âgé notamment par MADSEN (I963), NEHRING et al. (I963), HFinRY (I 668), mais peuvent s'expliquer par la différence observée en ce qui concerne la fréquence des diarrhées ; diminuée par l'orge et augmentée par le blé. 
Le porcelet sevré se comporte différemment du porc plus âgé vis-à-vis du constituant de base de sa ration. Les résultats des auteurs précédents se rapportent à la céréale à des doses très élevées dans le régime et de ce fait, à des rations variables en ce qui concerne le taux de cellulose et l'équilibre azoté. De plus, le manioc améliore de façon significative 1'utilisation du régime distribué soit à volonté, soit en quantité égalisée : l'amélioration varie de 3 à $5 \mathrm{p}$. Ioo pour les éléments majeurs du régime (protéines, énergie). Ces propriétés particulières du manioc ont déjà été soulignées par 'TERROINE (I93I), Jacquot et NATAF (I936). D'autres données confirment sa très bonne digestibilité chez 1'Homme (LANGworthy et DeUEI, I922), chez le Rat (Púrisse et Jacquot, I956), et chez la Volaille (Vogt et Stute, I964). Chez le Porc, seuls Homb et Lyso (I954) ont montré que le manioc introduit dans une ration à base d'orge en atgmente la digestibilité (matière organique et protéines), en accord avec nos résultats.

Les chiffres obtenus pour le maïs et 1'avoine décortiquée varient suivant le mode de distribution du régime. Lorsque les animaux sont alimentés à volonté, la digestibilité est significativement plus faible que pour le blé et peut s'expliquer en accord avec les résultats de Hvidsen et al. (I964) par un état sanitaire défectueux. Par contre, lorsque les aliments sont distribués en quantité limitée, les résultats ne sont pas significativement différents.

D'une façon générale, les valeurs obtenues en cage de digestibilité sont en bon accord en ce qui concerne la digestibilité de l'énergie avec celles obtenues par DIGGS et al. (I 965) sur le Porcelet, mais différentes de celles de MadSen (I963), Nehring (I963), Robinson et al. (I965) ou Henre (I968) sur le Porc en croissance et de celles de VeRMOREL, et KELLER (I967) sur le Rat.

I1 convient de plus de souligner l'identité des valeurs des coefficients de digestibilité, suivant le sexe des animaux ce qui permet sur le plan méthodologique d'utiliser un plus grand nombre d'animaux de la même portée pour les études de digestibilité. D'autre part, on observe une forte variation de l'utilisation digestive des éléments du régime aussitôt après le sevrage (fig. 3). De telles variations ont déjà été observées par Combs et al. (Ig63) et PEKas et al. (1964). Ainsi, il apparait qu'il est seulement possible de calculer une valeur moyenne pour les éléments digestibles d'un régime pour le Porcelet à partir de 6 semaines d'âge.

Dans nos conditions expérimentales, on peut remarquer que l'énergie digestible varie beaucoup plus suivant la nature du constituant de base qu'en fonction de la teneur en énergie brute du produit. Ainsi pour le Porcelet, les résultats peuvent être influencés par l'aptitude du régime, et en particulier par les propriétés spécifiques de certains amidons, à maintenir un bon état sanitaire chez les animaux (BAKER et al., I950). II en résulte que l'orge et le blé ont une valeur en énergie digestible identique, le maïs et l'avoine décortiquée, une valeur supérieure de moins de $2 \mathrm{p}$. Ioo alors que le manioc a une teneur supérieure au blé et à l'orge de plus de 5 p. Ioo.

Ces valeurs de l'énergie digestible sont tout à fait comparables à celles de DIGGS et al. (1965) pour le mais. Elles sont légèrement plus élevées pour le blé mais sensiblement plus faibles pour l'avoine décortiquée contrairement aux résultats de RICHTER et al. (I962) pour une avoine nue ou de Thomke (I960) pour une avoine décortiquée distribuée à des pores plus âgés.

Enfin, les valeurs trouvées pour les protéines digestibles du régime sont peu variables, ce qui peut s'expliquer du fait de la haute valeur biologique du complément 
protidique utilisé (tab1. I) qui apporte les $2 / 3$ de la ration azotée du porcelet. Dans ces conditions, on observe des coefficients de rétention azotée peu variables d'un régime à l'autre (tab1. 7), sauf pour l'avoine décortiquée en raison probablement du bon équilibre en acides aminés indispensables de ses protéines.

\section{Croissance des animaux et utilisation des aliments}

Les variations des performances pondérales des porcelets et de l'efficacité alimentaire globale sont en grande partie expliquées par les résultats de digestibilité et l'état sanitaire des animaux.

Ainsi, les régimes à base de manioc et d'orge permettent une vitesse de croissance maximum car ils entraînent une augmentation de la digestibilité apparente des éléments de la ration en même temps qu'ils provoquent une augmentation de la quantité moyenne d'aliment consommée par rapport au régime témoin (fig. I). Dans ce dernier cas, il semble bien que la consommation d'aliment soit fonction non plus de l'appétibilité propre de la céréale (SALMON-LEGAGNEUR et FÉVRIÉr, I959), mais de la tolérance de l'animal vis-à-vis du constituant de base de la ration. L'avoine décortiquée provoque globalement une amélioration des performances, mais les animaux doivent s'adapter à un tel régime qui est mieux utilisé lorsqu'un rationnement est pratiqué et la fréquence des diarrhées limitée (fig. 2). On observe en fin une amélioration de l'efficacité alimentaire, des efficacités protidique et énergétique des aliments lorsque les quantités offertes aux animaux sont égalisées par rapport au témoin et par conséquent limitées (exp. II, tabl. 8). Dans ce cas, on voit apparaître l'effet propre du constituant de base (céréale ou manioc) sur les performances du porcelet.

Ainsi, quelle que soit la méthode de distribution de l'aliment, l'effet du constituant majeur se traduit soit par une modification de la quantité consommée associée à une augmentation de l'utilisation de l'aliment, soit par une nette amélioration de l'utilisation de l'aliment qui provoque une variation des performances de croissance suivant le mode de distribution de l'aliment.

\section{Choix des éléments de base des régimes de sevrage}

Le choix des constituants de base de la ration de sevrage du porcelet doit tenir compte d'un certain nombre de critères parmi ceux envisagés précédemment (AUMAITRE, I967). Le meilleur régime doit concilier les impératifs physiologiques (facilité d'adaptation, limitation de la diarrhée), nutritionnels (efficacité alimentaire et digestibilité de la ration), et économiques (prix de revient minimum de l'aliment, vitesse de croissance maximum).

Si l'on tient compte des critères physiologiques et nutritionnels, le manioc doit être préféré ; l'orge paraît également intéressante ainsi que le blé. Par contre, en dépit de certains avantages (appétibilité, vitesse de croissance), 1'avoine décortiquée doit être utilisée en faible quantité en raison de son prix et du risque de mauvaise conservation ; l'utilisation du maïs comme seul élément de base ne semble pas non plus à conseiller. 


\author{
SUMMARY
}

\title{
NUTRITIVE VALUE OF MANIOC AND DIFFERENT CEREALS IN EARIY WEANING DIETS FOR THE PIGLET : DIGESTIVE UTILIZATION OF FEED AND ITS EFFECTS ON GROWTH OF THE ANIMALS
}

The effect of the type of basic component in the ration (manioc, cereals) has been studied in the early weaned piglet.

In two experiments, the effect on growth rate, feed efficiency, and digestibility of the main elements in the ration were measured when barley, manioc, decorticated oats and corn were introduced in proportion to wheat.

There was a significant effect on growth and feed consumption. Maximum performances were obtained with manioc and barley (4I6 and $386 \mathrm{~g}$ of weight gain/day) as against $360 \mathrm{~g}$ for wheat. These results may be cxplained as a special effect on the sanitary condition of the animals (decrease in the frequency of diarrhea).

The apparent digestibility of all the elements in the diet varied, depending on the kind of cereal (especially when the animals were full-fed). Thus, contrary to what has been observed in the growing pig, barley, corn and wheat were comparatively digestible when animals were pairedfed. Manioc improves digestibility by $4-5$ p. Ioo in relation to wheat, and decreases the frequency of diarrhca after weaning.

The over-all results show that wheat, barley and decorticated oats have comparable digestible encrgy value when associated to the same complementary feed (about 4 ooo $\mathrm{kcal} / \mathrm{kg}$ of $\mathrm{dry}$ matter), and that manioc is more energetic (about $4200 \mathrm{kcal}$ ).

\section{RÉFÉRENCES BIBLIOGRAPHIQUES}

Anowyme, I962. Tapiokamehl ein sehr gefragter Artikel. Schweinezucht Schreinemast., 10-19.

Aumaitre A., Jouandet C., Salmon-Legagneur E., I964. Étude des activités amylolytiques chez le porcelet en cours de sevrage. Ann. Zootech., 13 (H.-S.), 55-72.

Aumaitre A., I966. Étude de la dégradation enzymatique de divers amidons par le suc pancréatique de porcelet en cours de sevrage. Proc. VIIth. Int. Cong. Nutr. Hamburg, Pergamon Press. 5, 100-I03.

Aumaitre A., I967. Futterverwert von Tapioka und verschiedenen Getreidearten in Futterrationen für frühzeitig abgesetzte Ferkel. Zeit. Tierphysiol. Tievernähr. Futtermittelk., 23, 41-43.

Baker F., Nasr H., Morrice F., Bruce J., 1950. Bacterial breakdown of structural starches and starch products in the digestive tract of ruminant and non ruminant mammals. J. Path. Bact., 62, 6 I $7-638$.

Carroll W. IE., Krider J. L., I942. Swine Production. Mcgraw Hill Co. New York.

Combs G. E., Osegueda F. L., Wallage H. I), Ammerman C. B., I963. Digestibility of rations containing different sources of supplementary protein by young pigs. J. Anim. Sci., 22, 396-398.

Diggs B. G., Becker D. E., Jensex A. H., Norton H. W., I 965 . Energy value of various feeds for the young pig. J. Anim. Sci., 24, 555-558.

Dunnett C. W., I964. New tables for multiple comparisons with a control. Biometrics, 20, 482-491.

Guilbot A., Mercier Ch., ig62. Répercussions sur la digestibilité de l'amidon, des modifications de sa structure physico-chimique au cours de ses transformations technologiques. Inds. Aliment. Agric., 79, $939-947$.

Hansen V., I964. Tapiokamel til slagterisvin. Ugeshift Landmaend, 35, 562-563.

Hartmann P. A., and al. 1961. Digestive enzyme development in the young pig. J. Anim. Sci., 20, II 4-I23.

HENRY Y., 1968. Utilisation comparée des céréales comme seuls aliments du porc pendant la période de finition. Ann. Zootech., 17, I83-197.

Номв T., Lysö A., Ig64. Nitrogen balance experiments with growing pigs fed ground barley and sorghum grains. Meld. Norges Landbrugshögskole, 43, I-2 I.

Hvidsten H., Langebrekke A., Lynch G., Homb T., Ig64. Some results from preliminary norwegian experiments on baby pig nutrition. Ann. Zootech., 13 (H.-S.), 215-2I9.

Jacquor R., Nataf B., I936. Le manioc et son utilisation alimentaire. Hermann et Cie, Éd. Paris. 
Jouandet C., Aumaitre A., Salmon-Legagneur E., 1964. Influence des antibiotiques sur la croissance des porcelets sevrés à 5 semaines. Ann. Zootech., 13 (H.-S.), II3-I 27.

Langworthy C. F., DeUel H. J., x920. Digestibility of raw corn, potato and wheat starches. $J$. Biol. Chem., 42, 27-39.

Langworthy C. F., Deuel H. J., I922. Digestibility of raw rice, arrowroot, canna, cassava, taro, tree-fern, and potato starches. J. Biol. Chem., 52, $25 \mathrm{I}-26 \mathrm{I}$.

Lewis C. J. al. I955. Enzyme supplementation of baby pig diets. J. Agric. Food. Chem., 3, I047-I050

MADSEN A., I963. Digestibility experiments with pigs. Beretning Forsöglaboratoriet, 38\%, I-I9I.Köbenhavn.

Nehring K., Hoffmann L., Schiemann R., ig63. Die energetische Verwertung der Futterstoffe. 3 Die energetische Verwertung der Kraftfutterstoffe durch Schweine. Arch. Tierernähr, 13, 147-I61.

Pekas J. C., Hays V. W., Thompson A. M., 1964. Exclusion of the exocrine pancreatic secretion. Effect on digestibility of soyabean and milk protein by baby pigs at various ages. J. Nutr., 82, 277-284.

Périsse J., Adrian J., Jacouot R., r956. Étude in vivo et in vitro de la digestibilité du manioc sous différentes formes : farine entière, farine blutée, fécule et gary. Ann. Nutr. Alim., 10, I3-2 I.

RADLEY J. A., I953. Starch and its derivatives. Chapman and Hall, ed. London.

Richter K., Cranz K. L., Oslage H. J., I962. Hafer in der Tierernährung. Zeit. Das wirtschafteigene Futter., 1, I-55.

Robinson D. W., Prescott J. H. D., Lewis D., I965. The protein and energy nutrition of the bacon pig. IV. Digestible energy values of cereals in pig diets. J. Agric. Sci, 64, 59-66.

Salmon-Legagneur E., Février R., i959. Les préférences alimentaires des porcelets. III. Appétence de quelques céréales. Ann. Zootech., 8, 139-146.

Smith H., Lucas I. A. M., I957. The early weaning of pigs. III. The influence of protein supply during two stages of growth in the performance of pigs from glb to bacon weight. J. Agric. Sci., 49, 409-4I 7 .

Terroine E. F., I93I. De l'emploi des laits artificiels dans l'élevage du bétail. Bull. Soc. Sci. Hyg. Aliment. Paris, 19, I-23.

Thivend P., Mercier C., Guilbot A., ig65. Dosages de l'amidon dans les milieux complexes. Ann. Biol. anim. Biochim. Biophys., 5, 513-526.

Тномке S., I960. Studies on the digestibility of oats by pigs. Kungl. Landbrukshögskolans. Ann., 26, 269-288,

Vermorel M., Keller J., I967. Utilisation énergétique, par le rat en croissance, des principales céréales composant des régimes isoazotés et équilibrés en acides aminés. Ann. Zootech. 16, 223-234.

VoGt H., Stute K., I964. Prüfung von Tapiokapellets im Geflügelmast alleinfutter. Arch. Geflügelkde, 78, 342-358.

WALKER D. M,, I959. The development of the digestive system of the young animal. II. Carbohydrase enzyme development in the young pig. J. Agric. Sci., 52, 357-370.

Weijers H. A., Kamer J. H. van de, 1965. Alteration of intestinal bacterial flora as a cause of diarrhwea. Nutr. Abstr. Rev, 35, 591-604. 